\title{
Emerging Neuropathological and Acid-base Disorders in Coronavirus Disease-19: A Close Look at Diagnostic Prospect in Containment Operations
}

\author{
Kamoru Ademola Adedokun* (D) \\ Department of Oral Pathology, King Saud University Medical City, Riyadh, Saudi Arabia
}

\begin{abstract}
Edited by: Mirko Spirosk Citation: Adedokun KA. Emerging Neuropathological and Acid-base Disorders in Coronavirus Disease-19: A Close Look at Diagnostic Prospect in Containment Operation Open Access Maced J Med Sci. 2020 Oct 03; 8(T1):387-390 https://doi.org/10.3889/oamjms.2020. 5303 Keywords: Acidosis; Olfactory dysfunction; Asymptomatic; Coronavirus disease-19; Diagnostic utility; Severe acute respiratory syndrome coronavirus-2 Correspondence: Kamoru Ademola Adedokun Medical City, Riyadh, 11545 , Saudi Arabia
Mel: + 9662552442026 . E-mail: adeolokun@yahoo com Received: 24-Jul-2020 Revised: 11-Sep-2020 Accepted: 24-Sep-2020 Copyright: ๑) 2020 Kamoru A. Adedoku Funding: Publication of this article was financially pported by the Scientific Foundation SPIROSKI, Skopje, Republic of Macedonia Competing Interests: The authors have declared that no
competing interests exist Competing Interests: The authors have declared that no
competing interests exist under the terms of the Creative Commons Attribution-

The two major barriers militating against rapid containment of the spread of coronavirus (CoV) disease (COVID)-19 include lack of effective contact tracing and the failure to detect and diagnose the infection early. Lack of diagnostic tools for early diagnosis has contributed to the bane of the current wild spread of COVID-19 and its containment. The current chest computed tomography (CT) for COVID-19 screening, an evolving technique, is arguably reported to have $97 \%$ diagnostic sensitivity over the viral polymerase chain reaction (PCR) that has detection of $70 \%$. However CT has largely been criticized as speculative and thus generates disagreement among various international radiology societies and organizations. Until now, nucleic acid detection by real-time PCR (advanced with next-generation sequencing) remains the gold standard test and clinical diagnosis technique for COVID-19. The use of this method in diagnoses, while it is more precise, is also time-consuming and may not meet the goal of rapid detection of early infection with severe acute respiratory syndrome CoV-2. Although many available tests, such as other PCR-based, serology, isothermal nucleic amplification, and among others, are coming up, the testing accuracy and/or timeliness have hampered their expected performance level. As a result, there is still a need to develop more methods to detect the current spread of COVID-19 rapidly. COVID-19 is now associated with olfactory dysfunctions in several reports. Recently, the Centers for Disease Control (CDC) established that anosmia is a notable symptom of COVID19. Furthermore, acute systemic acidosis has been associated with COVID-19. This report critically discusses the potential pathophysiologies of COVID-19 in association with neuropathological and acid-base disorders and their prospect for diagnostics.
\end{abstract}

\section{Highlights of the Study}

Detection of early cases of COVID-19 remains a big concern against the containment operation

- This report discusses the pathophysiology of a neuropathological disorder, anosmia, as a possible diagnostic tool

- It also discusses the pathophysiology and potential diagnostic use of an associated acidbase disorder in COVID-19 patients

- The report concludes that putting apparatus, such as respiratory or smelling testing panels, into use could add to the benefit of early detection operation.

\section{Introduction}

The two major barriers militating against rapid containment of the spread of coronavirus (CoV) disease 2019 (COVID-19) include lack of effective contact tracing and the failure to detect and diagnose the infection early. Lack of adequate diagnostic tools for early diagnosis has impacted the current wild spread of COVID-19 and its containment. Extensive studies are ongoing to address these problems, especially for asymptomatic and presymptomatic patients. Majority of COVID-19 cases (81\%) show mild or no symptoms [1]. Unfortunately, these individuals may shed the virus and fuel the contagion. It becomes a matter of urgency to develop and improve on rapid diagnostic tools to aid early detection of severe acute respiratory syndrome CoV 2 (SARS-CoV-2) infected individuals, who are oftentimes asymptomatic before they can spread the disease. It is believed that asymptomatic spread has been aggravating the pandemic control of COVID-19.

In search of rapid techniques for detection of early infection with SARS-CoV-2, the chest computed tomography (CT) for COVID-19 screening has been found to detect $97 \%$ of COVID-19 cases and rapidly within 10 mins, while viral polymerase chain reaction only detects $70 \%$ sensitivity [2]. However, $97 \%$ sensitivity report with CT screening has been largely criticized as speculative and shows a lack of consensus among various reputable societies such as the American College of Radiology, Royal College of Radiologists, Royal Australian and New Zealand College of Radiology, and Canadian Association of Radiologists [2]. Recently, millions of antibody test kits ordered by UK governments in the wake of an urgent 
call to detect asymptomatic and presymptomatic patients and to prevent the rapid transmission of COVID-19 were not consistent and, thus, unreliable. Likewise, millions of test kits purchased by Slovakia, Czech Republic, and Spain from China were largely inaccurate [3].

Meanwhile, COVID-19 draws myriads of signs and symptoms with related pathogeneses. Of recent, the CDC established six more symptoms to the list of COVID-19 symptoms, including anosmia [4]. Up till date, the report has been associating anosmia/hyposmia, a neuropathological disorder with COVID-19 [5], the pathogenesis of which has not been clearly identified. Although an earlier study conducted retrospectively in Italy through a self-report identification method shows a low incidence rate of $19.4 \%$ [6]. In general, incidence rates of olfactory dysfunction (OD) in COVID-19 patients ranging between $33.9 \%$ and $68 \%$ [7] from further studies showed increased discovery rates. In other words, as time evolved with more research studies intensified toward containment of COVID-19, various investigations involving larger sample sizes, and/or more robust techniques show continued to show higher prevalence rates with the cases of OD becoming more evident and gradually taken into clinical considerations [8-11]. Of note is a multicenter-study that later reported an $85.6 \%$ prevalence rate with larger coverage, including many European countries such as Belgium, France, Spain, and Italy using questionnaire method [8]. Remarkably, a study conducted in Iran with the aid of a validated method using a 40-odorant test (University of Pennsylvania Smell Identification Test, method) reported about $98 \%$ smell dysfunctions proposing that ODs would serve as a key indicator to identify SARS-CoV-2-infected patients [10]. In addition, apart from OD, the respiratory disorder is generally associated with ventilation problems and a possible etiology of acute and chronic systemic acidosis in some pulmonary affected diseases. This report discusses the pathophysiologies of neuropathological and acidbase disorders in relation to olfactory dysfunction and systemic acidosis and their prospect in the diagnoses of COVID-19 and population surveillance.

\section{Emerging Neuropathological Disorder in COVID-19 and its Diagnostic Prospect}

Report from the experts in rhinology shows that, currently, there are large numbers of patients who tested for COVID-19 infection and developed anosmia/hyposmia in South Korea, China, Germany, Iran, US, France, and Italy [5]. The report further explains that majority of these patients present with anosmia even without other symptoms [5]. Recently, the CDC enlisted six more to the tally of COVID19 symptoms [4]. It is believed that certain forms of OD may be impacted in anosmia/hyposmia. Post-viral olfactory disorder has been described as one of the possibilities in developing anosmia [12]. The exact mechanism of olfactory neurobiology behind the development of post-viral anosmia in COVID-19 has not been reported. Nonetheless, two possible etiologies may be associated with the apparent loss of smell. The first probable etiology could be related to loss of maintenance or replacement of olfactory neuron as a result of post-viral damage in relation to the reduction of basal stem cell in the olfactory neuroepithelium. Another but different mechanistic plausibility is immunobiological which may be related to constant inflammatory post-viral damage that may have impaired either or both olfactory neuron and basal cell function. In a related study, early data show that provoked inflammatory reactions (implicating some inflammatory cytokines, such as interleukins- 6 and $1 \beta$ ) occur in both upper and lower respiratory tracts following infection with SARS-CoV-2 with consequent respiratory problems ranging from mild to extensive life-threatening lung injury [13].

While in search of simple and sensitive detection diagnostic or screening tools, the development of smell testing kits and/or symptom diagnostic criteria may offer significant testing opportunities in this area of COVID-19 pathology. In clear terms, the development of symptom diagnostics may involve identifying vital differentials that will clearly indicate loss of smell, specifically in relation to postviral infection with SARS-CoV-2, following the exclusion of unrelated pathological disorders that may range from chronic rhinitis to neurodegenerative processes. In addition to testing diagnostic kits, the development of OD symptom diagnostic criteria may add values to early detection of COVID-19 in combination with the existing diagnostic techniques for various OD manifestations. It is believed that the use of simple odorant testing kits and symptom diagnostic criteria could reduce the time spent on laboratory diagnoses and possibly aid in early detection or screening process during containment operations.

\section{CoVID-19 with an Associated Acid-base Disorder and the Diagnostic Utility}

Apart from asymptomatic cases, several individuals with mild symptoms or presymptomatic may also escape quarantine control measure and continue to spread the virus through several contacts. It may be essential to develop screening panels or diagnostics in relation to change in body physiology. The successful development of screening panel and rapid diagnostic tools required for the detection of COVID-19 suspected cases have been hampered due to complex mechanisms of pathogenesis of the disease that underlie the development of signs and symptoms. Besides, signs and symptoms in patients suffering from COVID-19 are diverse in range and this also contributes against the development of effective rapid diagnostics. Pathophysiologically, the development of signs and symptoms is generally a result of alteration 
in body physiology. In other words, the development of signs and symptoms has clinical correlations with changes in body function. Alteration in body chemistry could also serve an advantage to develop screening or detection panel to support early diagnosis.

Cases of COVID-19 have been reported with the involvement of multiple organs. Likewise, SARSCoV-2 has been detected in blood, feces, and urine samples [14]. It is not certain whether SARS-CoV-2 is transported through a lymphohematogenous route to other organs similar to other agents of pulmonary infection that has been reported elsewhere in distal body tissues [15]. However, the common signs and symptoms in COVID-19 are particularly related to respiratory problems. Clinical manifestations relating to respiratory symptoms such as cough, dyspnea (shortness of breath), runny nose, and other secondary effects of a respiratory problem involving tachycardia, hypoxia, acidosis, and among other findings have been reported [14], [16]. Respiratory acidosis is a complication of breathing problem. In the buildup of body acidosis, there is a reduction in arterial body $\mathrm{pH}$ and stimulation of peripheral chemoreceptors that result in increased ventilatory drive under body regulation through a compensatory mechanism. As described in Figure 1, the presence of an obstructive gas exchange body tends to increase more $\mathrm{CO}_{2}$ in the body in consequence of alveolar hypoventilation that drives respiratory acidosis due to the creation of an acidic environment. Alveolar hypoventilation leads to an increased $\mathrm{PaCO}_{2}$. Ventilatory defect that leads to the acid-base disorder may offer useful diagnostic information in identifying suspected cases of COVID-19. Estimation of changes in arterial $\mathrm{pCo}_{2} / \mathrm{pO}_{2}$ can be done through a breathing record of inspired and expired gasses using various respiratory gas measurements and thus stands a rapid advantage to pave new screening or diagnostic opportunities.

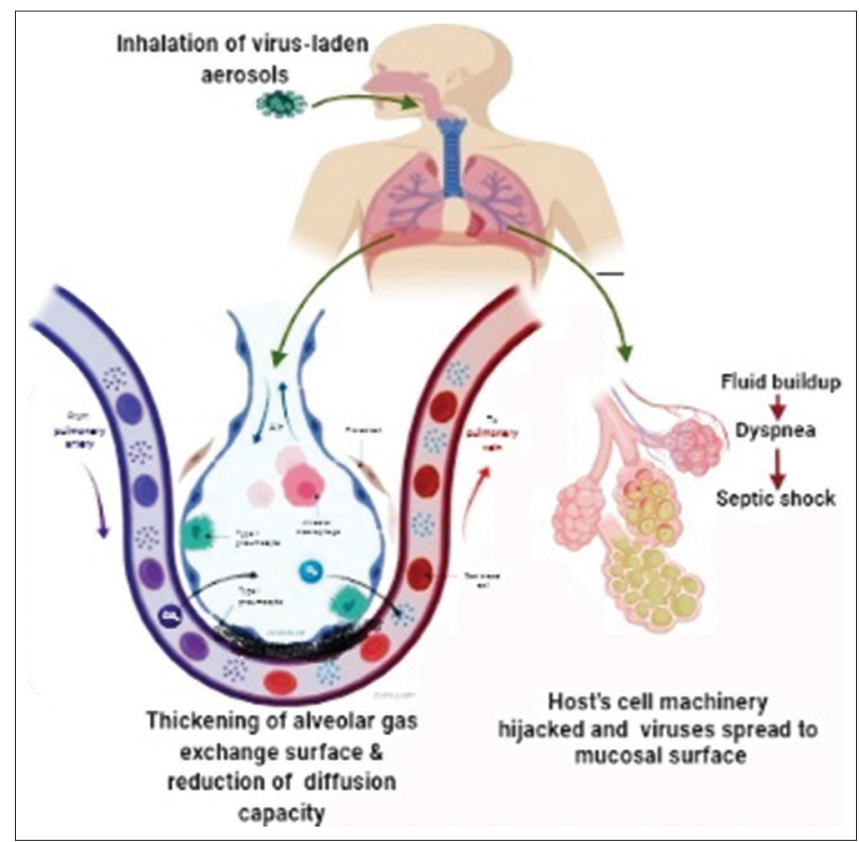

Figure 1: Development of acute respiratory distress in critical cases associated with COVID-19

\section{Conclusion}

Knowledge of the changes in body physiology and/or chemistry from the available clinical information in relation to the pathophysiologies of associated neuropathological and acid-base disorders in COVID19 may help in better understanding the disease and also aid the development of screening or diagnostic tests. Perhaps, this may circumvent waiting long for laboratory analyses or development of biomarkers or add to the diagnostic efficiency of COVID-19. Thus, the development of respiratory virus testing panels that are highly sensitive and specific for SARS-CoV-2 target may help in containment operation through the detection of the hidden carriers who are asymptomatic or presymptomatic.

\section{Declarations}

\section{Ethical approval}

Ethical approval and written informed consent not required for this study.

\section{Consent for publication}

No data in this study required written informed consent before publication.

\section{Availability of data and material}

Data sharing is not applicable; no dataset was generated or analyzed during the present study.

\section{References}

1. Wu Z, McGoogan JM. Characteristics of and important lessons from the coronavirus disease 2019 (COVID-19) outbreak in China: Summary of a report of 72314 cases from the Chinese center for disease control and prevention. JAMA. 2020;323(13):1239-42.

PMid:32091533

2. Ai T, Yang Z, Hou H, Zhan C, Chen C, Lv W, et al. Correlation of chest CT and RT-PCR testing for coronavirus disease 2019 (COVID-19) in China: A report of 1014 cases. Radiology. 2020;296(2):E32-40.

PMid:32101510

3. Loh T. Available from: https://www.bloomberg.com/news/ articles/2020-04-07/new-test-hopes-dashed-as-u-k-findsantibody-kits-don-t-deliver. [Last accessed on 2020 Mar 19].

4. CDC 2020. Symptoms of Coronavirus; 2020. Available from: https://www.cdc.gov/coronavirus/2019-ncov/symptoms-testing/ symptoms.html. [Last accessed on 2020 Mar 19]. 
5. ENT UK; 2020. Available from: https://www.entuk.org/losssense-smell-marker-covid-19-infection. [Last accessed on 2020 Mar 19].

6. Vaira LA, Salzano G, Petrocelli M, Deiana G, Salzano FA, De Riu G. Validation of a self-administered olfactory and gustatory test for the remotely evaluation of COVID-19 patients in home quarantine. Head Neck. 2020;42(7):1570-6. https://doi. org/10.1002/hed.26228

PMid:32357379

7. Meng X, Deng Y, Dai Z, Meng Z. COVID-19 and anosmia: A review based on up-to-date knowledge. Am J Otolaryngol. 2020;41(5):102581.

PMid:32563019

8. Lechien JR, Chiesa-Estomba CM, De Siati DR, Horoi M, Le Bon SD, Rodriguez A, et al. Olfactory and gustatory dysfunctions as a clinical presentation of mild-to-moderate forms of the coronavirus disease (COVID-19): A multicenter European study. Eur Arch Otorhinolaryngol. 2020;277(8):2251-61. https://doi. org/10.1007/s00405-020-05965-1 PMid:32253535

9. Luers JC, Rokohl AC, Loreck N, Matos PA, Augustin M, Dewald F, et al. Olfactory and gustatory dysfunction in coronavirus disease 19 (COVID-19). Clin Infect Dis. 2020:ciaa525. https://doi. org/10.1093/cid/ciaa693

PMid:32357210

10. Moein ST, Hashemian SM, Mansourafshar B, Khorram-Tousi A,
Tabarsi P, Doty RL. Smell dysfunction: A biomarker for COVID19. Int Forum Allergy Rhinol. 2020;10(8):944-50. https://doi. org/10.1002/alr.22587

PMid:32301284

11. Yan $\mathrm{CH}$, Faraji F, Prajapati DP, Ostrander BT, DeConde AS Self-reported olfactory loss associates with outpatient clinical course in COVID-19. Int Forum Allergy Rhinol. 2020;10(7):82131. https://doi.org/10.1002/alr.22592

12. Goncalves S, Goldstein BJ. Pathophysiology of olfactory disorders and potential treatment Strategies. Curr Otorhinolaryngol Rep. 2016;4(2):115-21.

13. Conti P, Ronconi G, Caraffa A, Gallenga CE, Ross R, Frydas I, et al. Induction of pro-inflammatory cytokines (IL-1 and IL-6) and lung inflammation by coronavirus-19 (COVI-19 or SARSCoV-2): Anti-inflammatory strategies. J Biol Regul Homeost Agents. 2020;34(2):327-31.

PMid:32171193

14. Cascella M, Rajnik M, Cuomo A, Dulebohn SC, Di Napoli R. StatPearls. Treasure Island, FL: StatPearls Publishing; 2020.

15. Eziyi AK, Oluogun WA, Adedokun KA, Oyeniyi GA. Prostate tuberculosis: A rare complication of pulmonary tuberculosis with malignant features mimicking prostate cancer. Urol Sci. 2020;31:36-8.

16. Adedokun KA. Early stage nonclinical pulmonary disorder in COVID-19 may present asymptomatic and fuel the contagion. Mil Med Res. 2020;2020:1-6. 